\title{
Comparative Analysis of Health Resources Allocation in Urban and Rural Hospitals of Jiangxi Province
}

\author{
Song Weicai ${ }^{1,}$ a, Wu Yanxia ${ }^{2, b}$ \\ ${ }^{1}$ College of Computer Science and Technology, Jiangxi University of Traditional Chinese Medicine, \\ Nanchang, China \\ ${ }^{2}$ College of Humanities, Jiangxi University of Traditional Chinese Medicine, Nanchang, China \\ asongweicai2000@sina.com; Corresponding Author: Wu Yanxia
}

Keywords: urban and rural hospitals; health resources; resource allocation

\begin{abstract}
Objective: Based on the analysis of the present situation of the urban and rural health resource allocation in Jiangxi Province, this paper provides scientific decision-making for the health administrative department. Methods: Using spss19.0, Lorenz curve and Gini coefficient, the data of urban and rural health resources in Jiangxi Province were analyzed from 2008 to 2014, and the statistical results were processed and analyzed. Results: The allocation of health resources in urban and rural areas is still a big gap, so we should strengthen the construction of grass-roots talent team, improve the level of medical services in rural hospitals, so that the allocation of health resources gradually reasonable.
\end{abstract}

\section{Introduction}

For a long time, due to the constraints of system and government investment, the development of urban and rural health in Jiangxi is not balanced, The health resources of urban hospitals are well distributed, while the rural hospitals are lack of health technical personnel, the technical level is low, the medical equipment is obsolete or inadequate, $\mathrm{T}$ Therefore, difficult to see a doctor, expensive, Jiangxi urban and rural residents has become a major problem ${ }^{[1-4]}$. Rational allocation of urban and rural medical and health resources, the development of urban and rural health services, the establishment of a new type of urban and rural health service system, is the key to solve the difficult and expensive to see a doctor. Using the Lorenz curve and the Gene coefficient to analyze the current situation of health resources allocation in urban and rural hospitals in Jiangxi Province during 2008-2014, and provide a scientific basis for the development of Jiangxi decision by the administrative department of public health.

\section{Materials and methods}

Source: This data is from the 2008-2008 "Jiangxi Health Statistics Yearbook".

Research contents: This paper makes a comprehensive analysis and Research on the urban and rural hospitals in Jiangxi province from 2008 to 2014, including beds, health technicians, registered nurses, and urban and rural residents.

Research methods: In this paper, a comparative study, statistical analysis, in addition to the allocation of health resources in urban and rural areas using Lorenz curve and the Gini coefficient method to determine fairness and justice. The Gene coefficient is based on the Lorenz curve reflects the statistical index, the degree of social equity of income distribution at present, equity evaluation of Gene coefficient has been widely used in health resource allocation, the basic steps are as follows: first, according to the ownership of resources per capita is to sort, then the population (or area) data and resource data into the corresponding mathematical formula ${ }^{[3]}$.

$$
G=\sum_{i=1}^{k}\left(P_{i} S_{i+1}\right)-\sum_{i=1}^{k}\left(P_{i-1} S_{i}\right)
$$

Where G represents the Gini coefficient, $\mathrm{Pi}$ is the cumulative percentage of population or area, $\mathrm{Si}$ is the cumulative percentage of health resources distribution. The value of Gene's system is 
between 0 and 1 , the closer to 0 , the more the income distribution tends to be equal.According to the relevant organizations of the United Nations: If the Gini coefficient is less than 0.2 , the income is absolutely average; $0.2-0.3$ is the average; $0.3-0.4$ is relatively reasonable; $0.4-0.5$ that the income gap is large; 0.5 above the income gap ${ }^{[5-7]}$. This paper describes the equity of health resources allocation in urban and rural hospitals in Jiangxi province by using Lorenz curve and the coefficient of Gene

\section{Results and analysis}

In this paper, statistical software SPSS19.0 was used to analyze the health resources of the urban and rural hospitals in Jiangxi province from 2008 to 2014, including the beds, health technicians, registered nurses and so on, as shown in Figure 1 to figure 4.

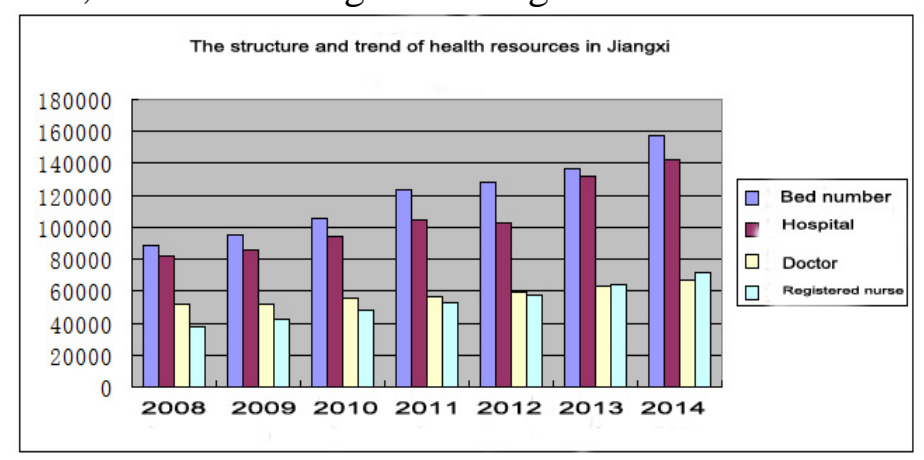

Figure 1

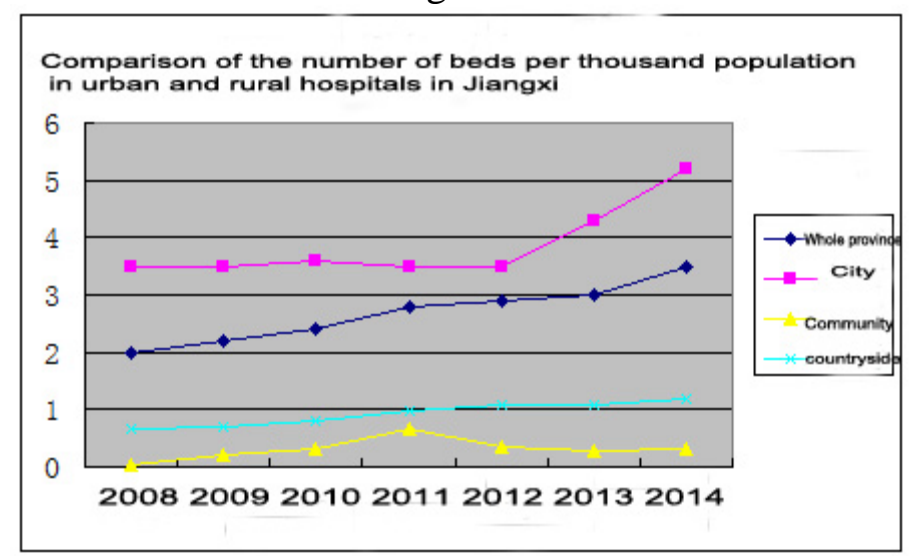

Figure2

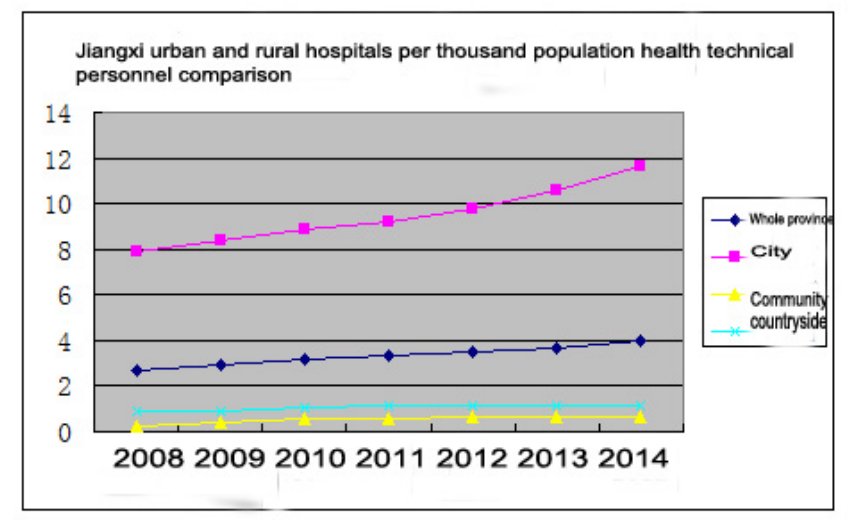

Figure 3

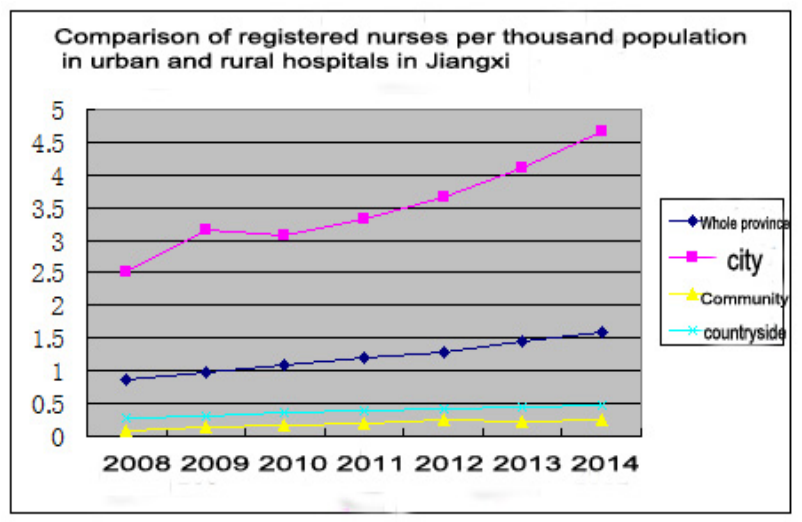

Figure 4

It can be seen from Figure 1, Jiangxi Province, a variety of health resources are growing steadily, the number of beds and hospitals, hospitals grew far faster than doctors and registered nurses growth rate. From 2008 to 2011, the number of doctors in Jiangxi Province than the number of nurses, this allocation of health resources is clearly unreasonable, according to the Ministry of Health requirements than the ratio of 1: 2, the number of nurses in Jiangxi is still far from enough, By the end of 2014, Jiangxi's health care ratio was only 1: 1.1, nurses also have a large gap ratio and 
rising space. Due to the shortage of nurses has two aspects, one is the majority of the hospital medical care light, the nursing staff in the hospital in the status and importance are ignored; two nursing staff is low treatment, resulting in a large number of nursing professionals drain. See from Figure 2, figure 3, figure 4, from 2008 to 2014 in Jiangxi province urban and rural community hospital hospital beds per thousand population, health technical personnel, registered nurses are basically a slow growth trend, while the city hospital beds per thousand population and health workers, but the growth rate of registered nurses very quickly, the cause of urban and rural growth trend of three aspects, first of all from the beginning of Jiangxi Province, health care reform, has been to town as the center, over time, The gap between medical resources and health level between urban and rural areas is more and more obvious; secondly, the human, material and financial resources of health care have long been focused on urbanization, which makes far more hygienic resources than urban areas. The duality of urban and rural areas, the duality of rights, the duality of rights and the duality of the subject, and the existence and expansion of the gap between the rich and the poor of urban and rural residents have led to the unequal distribution of medical and health resources in Jiangxi.

Next, this paper compares the urban and rural population allocation in Jiangxi in 2014 by Lorenz curve,see Figure 5 - Figure 7.

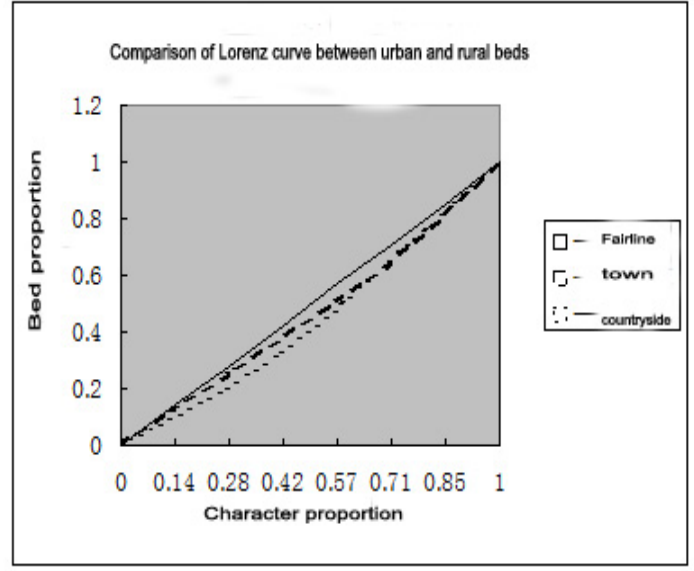

Figure 5

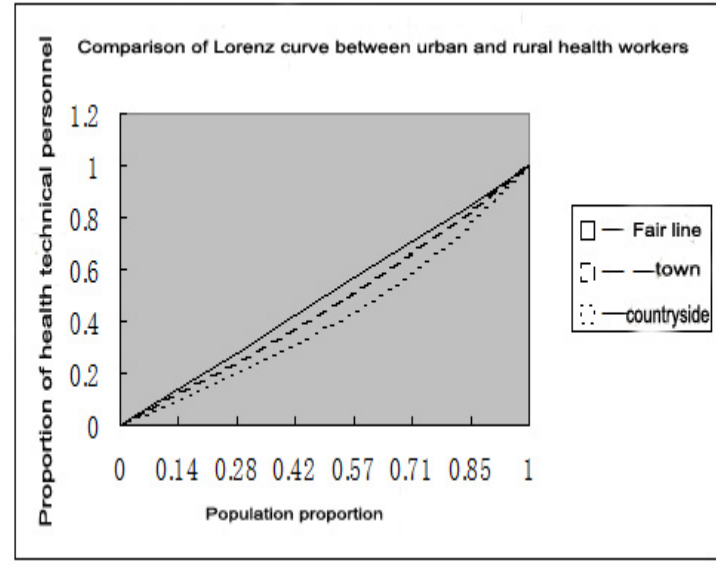

Figure 6

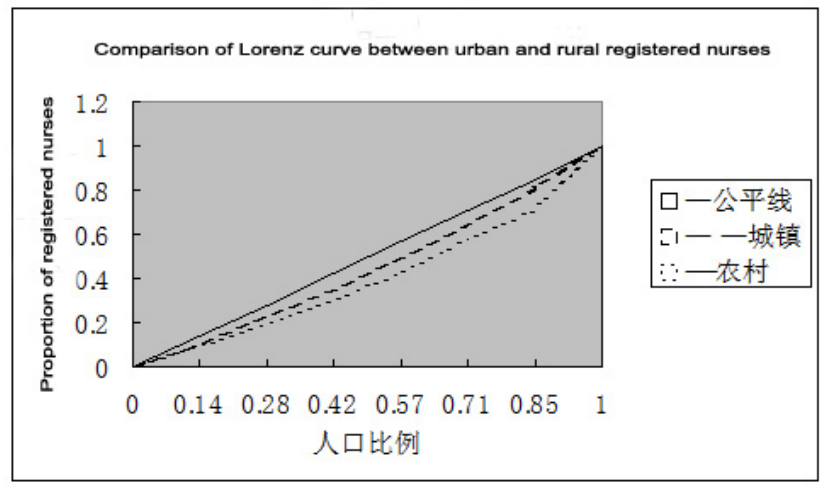

Figure 7

In the three graphs, the diagonal lines are solid lines, indicating the absolute fairness of the allocation of health resources, the long lines show the city health resources Lorenz curve, short dashed line that rural health resources Lorenz curve. From Figure 5, Figure 6, Figure 7 can be seen, the town of beds, health technicians, registered nurses Lorenz curve from the absolute fair line closer, indicating that the health allocation of urban hospitals more equitable and reasonable, and rural hospitals Of the beds, health technical staff, registered nurses Lorenz curve from the absolute fair line farther, indicating that the rural hospital health resources allocation is more unfair and more unreasonable. In order to further analyze the gap between the allocation of health resources in urban and rural hospitals, the Gini coefficient of beds, health technicians and registered nurses in urban and rural hospitals in 2008 to 2014 was compared. see Table1 
Table1.2008-2014Comparison of Gini coefficient between urban and rural health resources in Jiangxi

\begin{tabular}{|c|c|c|c|c|c|c|}
\hline \multirow{3}{*}{$\begin{array}{l}\text { year } \\
2008\end{array}$} & \multicolumn{2}{|c|}{ Bed Gini coefficient } & \multicolumn{2}{|c|}{ Gene coefficient of health technicians } & \multicolumn{2}{|c|}{ Gini coefficient of registered nurses } \\
\hline & \multicolumn{2}{|c|}{ town countryside } & \multicolumn{2}{|c|}{ town countryside } & \multirow{2}{*}{$\frac{\text { town }}{0.15}$} & countryside \\
\hline & 0.26 & 0.499 & 0.24 & 0.509 & & 0.502 \\
\hline 2009 & 0.24 & 0.403 & 0.23 & 0.501 & 0.15 & 0.500 \\
\hline 2010 & 0.24 & 0.384 & 0.23 & 0.489 & 0.14 & 0.493 \\
\hline 2011 & 0.21 & 0.373 & 0.21 & 0.475 & 0.13 & 0.485 \\
\hline 2012 & 0.20 & 0.354 & 0.21 & 0.443 & 0.11 & 0.473 \\
\hline 2013 & 0.19 & 0.351 & 0.18 & 0.432 & 0.10 & 0.461 \\
\hline 2014 & 0.19 & 0.345 & 0.17 & 0.427 & 0.07 & 0.447 \\
\hline
\end{tabular}

From Table 1 can be seen, the town hospital beds, health technicians, registered nurses Gini coefficient is small, and showed a downward trend, indicating that the various types of health facilities in urban hospitals more and more fair and reasonable. Rural hospital beds, health technicians, registered nurses have a larger Gini coefficient, exceeding the warning coefficient of 0.4 , or even more than 0.5 , which shows that the allocation of rural health resources are extremely unfair and extremely unreasonable, which also Means that rural residents can not enjoy the same as the urban residents, fair health services. From 2008 to 2014, the Gini coefficient of urban and rural health resources basically decreased, this is mainly because in recent years, with the development of urban and rural medical insurance system fully implemented in Jiangxi, urban and rural residents' demand for health care is unprecedented, resulting in 2008 - 2014 years of urban and rural hospital beds, medical and nursing staff ratio increased gradually however, a variety of health resources in rural hospitals increased much more slowly than urban hospitals, especially medical personnel and medical equipment and medical staff of the proportion of the growth experience, quality and technical level are far behind the urban hospital. This has resulted in many common diseases in rural township hospitals can not cure, the health resource allocation situation leads to more and more farmers in rural hospitals do not trust and go directly to the county hospital and city hospital, which directly leads to a variety of health resources in rural hospitals configuration is much slower in the town hospital.

\section{Discussion}

The allocation of health resources is a reasonable allocation of health resources in the area, so as to realize the fairness and justice of the allocation of health resources, and improve the efficiency of health resources. In order to achieve the rational allocation of health resources in Jiangxi, it is necessary to combine the internal and external environmental factors of the province, and adapt to it. On the one hand, Jiangxi is a mountainous province, rural residents in the province accounted for $73 \%$ of the total population, At present, the aging of Jiangxi is gradually accelerated, the proportion of the elderly and infants accounted for the proportion of the population of the province's $1 / 4$. On the other hand, with the acceleration of urbanization, the urban population is more and more big, therefore, in the formulation of health policy and allocation of health resources, should be a comprehensive analysis, to ensure that the allocation of health resources and the situation to adapt to the internal environmental factors which is now in Jiangxi, in the rational allocation of health resources in Jiangxi must first considering the internal environment, especially the elderly and infants with the proportion of the population is increasing year by year, it will increase the demand for health services across the province.

The rational allocation of health resources and health development needs to adapt to the trend, with the development of society, the needs of people's health is also rising, and health service are also people from the patient to the general health transition, change of medical mode and adapt to the change of the allocation of health resources, the only way to adapt to urban and rural residents 
more and more high demand for health. At present, Jiangxi's economic development level gradually rise in the central region, the allocation of health resources and Jiangxi should also adapt to the economic development level, the external environmental factors which is in Jiangxi, only the combination of internal and external environmental factors of Jiangxi, optimize the allocation of health resources to better serve the broad masses of the people in urban and rural areas.

\section{Acknowledgements}

The authors gratefully thank the financial support from Research on Humanities and Social Sciences in Jiangxi Province (GL1329).

\section{References}

[1] Song Weicai, He Yan, Wu Yanxia. Comparison and analysis of the input-output and medical benefits of various hospitals in Jiangxi Province. [J]. China Health Service Management .2011, (2): 114-116

[2] Song Weicai, He Yan, Wu Yanxia. Analysis of the input-output efficiency of two hospitals in Jiangxi Province.[J]. China health economic.2011 (6): 57-58

[3] Lai YouWen, Meng Qingyue, Wang Wenhua. Analysis on the equity of health resources allocation in three areas of Tibet [J]. China health economics, 2013, (1):63-64

[4] Luo Juan, Wang Hong, Cui Kaichang. Analysis of medical resources allocation in Shanghai [J]. Chinese Journal of Health Statistics, 2009, (5): 466-469

[5] Wang Wenying, Yuan Zhaokang, Yu Huiqiang. Investigation on human resources of community health service centers in Jiangxi [J]. Journal of Nanchang University (Medical Science), 2011, (7): 25-29

[6] Wang Wenying, Yuan Zhaokang, Yu Huijiang, Liu Yong. Investigation and Analysis on the infrastructure of urban community health service centers in Jiangxi [J]. Journal of Nanchang University (Medical Sciences) 2011, (8): 35-39

[7] Chen Dafu, Yu Kongying, Zhan Zequn. Analysis on the balance of human resource allocation of primary health care in Jiangxi Province Based on the new medical reform [J].. Chongqing medical journal, 2013, (11): 1312-1313 